\title{
Accurate Pipe Length Measurement Under Noisy Environment Using Stationary Waves
}

\author{
M. Okamoto, M. Nakayama and S. Tanaka \\ Faculty of Engineering, Yamaguchi University \\ 2-16-1 Tokiwadai, Ube, Yamaguchi, JAPAN \\ okamoto@sens.eee.yamaguchi-u.ac.jp
}

\begin{abstract}
The authors previously developed an accurate pipe length measurement system using stationary waves in pipes. For pipes with open ends in manufacturing fields, however, the system cannot always be directly applied, because noises from outside the pipes enter them at their open ends and disturb the stationary waves inside the pipes and cause successive phase shifts in the stationary waves.
\end{abstract}

The paper proposes a measurement system which realizes an accurate pipe length measurement under such a noisy environment by selecting the optimal data window and the optimal modes.

\section{Introduction}

In the field of measurement, we often encounter the situation where pipe lengths have to be indirectly measured such as in pipelines of gas, water and electric cables underground.

The authors therefore proposed previously a pipe length measurement system by making use of the stationary waves in the pipe generated spontaneously or by hitting it with a club $[1,2]$. In the system, sound fluctuations and their time derivatives of some stationary waves were adopted as the state variables of a linear dynamic system, and a Kalman filter and a maximum likelihood method were applied to the system to estimate the unknown parameters $L$ (pipe length) and $V$ (observation noisse variance).

Moreover, paying attention to the fact that high order modes of the stationary waves have generally a high resolution ability with respect to the measurement, the authors presented a selection criterion of the optimal modes to be used to model the dynamic system and realized a much higher accurate measurement of the pipe length[1].

However, in the case where the length measurement of open-end pipes is achieved in the place where an extremely large noise invades continuously into the pipe, such as in factories, an accurate pipe length measurement is difficult with the measurement system, because

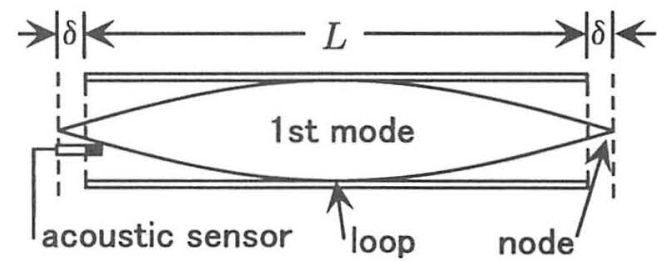

Fig. 1 Stationary waves in the pipe with two open ends.

the noise outside the pipe affects continually the amplitude and the phase of the stationary waves in the pipe.

To overcome the problem, the paper proposes a pipe length measurement system which gets rid of the affect by the noise. This is achieved by selecting the optimal data window and the optimal modes which are hardly influenced by the noise. Experimental results will show that an accurate length measurement of the pipes with open ends is achieved even in a noisy environment.

\section{Pipe length measurement by the stationary wave model}

We consider a straight pipe of length $L$ with two open ends as shown in Fig.1. Considering a short time interval of no noise, each mode of the stationary wave in the pipe is expressed as follows :

$$
x_{j}(t)=\alpha_{j} \sin \left(\omega_{j} t+\phi_{j}\right) \quad(j=1,2,3, \cdots)
$$

where $\omega_{j}$ stands for the angular frequency of the $j$-th mode of the stationary wave and is given by $\omega_{j}=$ $j \times 2 \pi v /\{2(L+2 \delta)\}$, where $v$ is the sound velocity in the pipe and $\delta$ an open end compensation[3]. Here, $\delta$ is given as $\delta=c r / 2$, where $r$ is the radius of the pipe and $c$ is the open end compensation coefficient.

We consider a set of mode numbers defined by $\Omega=$ $\left\{i_{1}, i_{2}, i_{3}\right\}$ and consider to approximate the sound fluctuation in the pipe $z(t)$ as a linear combination of these modes as follows:

$$
z(t)=\sum_{j \in \Omega} x_{j}(t)
$$


Then, defining the state vector $x=\left(x_{i 1}, \dot{x}_{i 1}, x_{i 2}, \dot{x}_{i 2}, x_{i 3}\right.$, $\left.\dot{x}_{i 3}\right)^{T}$, we obtain the following dynamic equation on the stationary waves of the modes.

$$
\dot{x}(t)=A x(t)+w(t)
$$

where

$$
A=\left[\begin{array}{ccc}
A_{1} & O & 0 \\
O & A_{2} & 0 \\
O & O & A_{3}
\end{array}\right], A_{j}=\left[\begin{array}{cc}
0 & 1 \\
-\omega_{i_{j}}^{2} & 0
\end{array}\right]
$$

where $\boldsymbol{w}(t)=\left(0, w_{1}(t), 0, w_{2}(t), 0, w_{3}(t)\right)^{T}$ represents a transition noise vector introduced to compensate for an attenuation of the stationary waves in the time interval. In our previous paper[1], the transition noise variances are already found hardly to affect the measurement accuracy. We thus assume that $w_{j}(t)(j=1,2,3)$ are mutually independent white Gaussian noises with means zero and variances $\sigma^{2}$.

Regarding unused modes of the stationary wave and the intrinsic sensor noise as the observation noise $v_{k}$, the observation equation (given by an acoustic sensor inside the pipe) is modeled as

$$
y_{k}=h x_{k}+v_{k} \quad(k=0,1,2, \cdots)
$$

where $\boldsymbol{h}=(1,0,1,0,1,0)$.

Consequently; the linear dynamic system of the stationary wave is obtained as (3) and (5). Descretizing the equations (3) and (5) with a sampling period, the estimation of the state vector is excuted by a discrete Kalman filter[4]. However, as the pipe length $L$ and the observation noise variance $V$ are unknown, Kalman filter cannot be directly applied.

Thus, defining the unknown parameters $L$ and $V$ as a vector $\theta=(L, V)^{T}$ and assigning adequate values, Kalman filter can be applied. Consequently, the pipe length measurement is achieved by maximization of the following likelihood function.

$$
\begin{aligned}
J(\boldsymbol{\theta}) & =p(\boldsymbol{y}, \boldsymbol{\theta}) \\
& =\prod_{k=1}^{K} p\left(y_{k} / \boldsymbol{\theta}, Y^{k-1}\right)
\end{aligned}
$$

where $p\left(y_{k} / \theta, Y^{k-1}\right)$ represents a conditional probability density function with respect to $y_{k}$ under the observation $Y^{k-1} \triangleq\left\{y_{j} ; 0 \leq j \leq k-1\right\}$ and the unknown parameter vector $\theta[1]$.

\section{Selection of the optimal data window and the optimal modes}

In our previous report, for any given data window, we gave a criterion to obtain the optimal modes to realize high accurate measurement. This criterion was to maximize the following Fisher's information matrix with respect to the modes[5].

$$
\begin{aligned}
K(L)= & \int\left[\frac{\partial}{\partial L} \ln p(\boldsymbol{y}, L)\right]\left[\frac{\partial}{\partial L} \ln p(\boldsymbol{y}, L)\right]^{T} \\
& \times p(\boldsymbol{y}, L) d y
\end{aligned}
$$

Under no external noise, high accurate pipe length measurement was achieved using the measurement system introduced in section 2 which used the optimal modes, despite the pipe end is closed or open. However, in the case where an extremely large noise continuously enters into the pipe, abrupt variations in the amplitude and phase of the stationary wave are caused. The variation of amplitude can be somewhat compensated by increasing the variance of the observation noise. But, the variation of phase causes a large deterioration in the measurement due to the shift of frequencies in the stationary wave. Therefore, not only the modes of stationary wave but also the data window have to be adequately selected to realize a high accurate length measurement for open pipes. It will be reasonable to consider here too to directly adopt the Fisher's infomation matrix for selecting both optimal data window and optimal modes. Instead of this, we adopt here the maximizing the sharpness of the likelihood function as the criterion. This is because the magnitude of the Fisher's information matrix corresponds to the secondary coefficient of the logarithmic likelihood function $\ln p(y, L)$ when the function is approximated by a quadratic function around the true value $L$. Below, we show the equivalence of the Fisher's information matrix and the sharpness of the peak of $\ln p(y, L)$. Considering a short time interval (i.e., a data window), the likelihood function can be given as :

$$
\begin{aligned}
p(\boldsymbol{y}, L)= & \frac{1}{(2 \pi)^{K / 2}|V|^{1 / 2}} \\
& \times \exp \left[-\frac{1}{2}(\boldsymbol{y}-\boldsymbol{\beta}(L))^{T} V^{-1}(\boldsymbol{y}-\boldsymbol{\beta}(L))\right]
\end{aligned}
$$

where $K$ is a number of data in the data window and $\boldsymbol{\beta}$ represents the following vector.

$$
\begin{aligned}
\beta & =\left(\beta_{1}, \beta_{2}, \cdots, \beta_{K}\right)^{T} \\
\beta_{k} & =\sum_{j \in \Omega} \alpha_{j} \sin \left(\omega_{j} k \Delta T+\phi_{j}\right)
\end{aligned}
$$

where $\omega_{j}$ represents the angular frequency of the $j$-th mode of the stationary wave and is defined as $\omega_{j}=$ $j \times 2 \pi v /\{2(L+2 \delta)\}(j \in \Omega)$. It is easily seen that $\beta$ becomes a function of $L$. Executing the Taylor expansion of the logarithm of the function (8) up to the second order around $L$, the following equation is ob- 
tained.

$$
\begin{aligned}
\ln p(\boldsymbol{y}, L)= & -\frac{1}{2}\left(\frac{\partial \boldsymbol{\beta}}{\partial L}\right)^{T} V^{-1}\left(\frac{\partial \boldsymbol{\beta}}{\partial L}\right)(\Delta L)^{2} \\
& +(\boldsymbol{y}-\boldsymbol{\beta}(L))^{T} V^{-1}\left(\frac{\partial \boldsymbol{\beta}}{\partial L}\right) \Delta L+\text { const. }
\end{aligned}
$$

On the other hand, substituting (8) into (7), the Fisher's information matrix is calculated as :

$$
\begin{aligned}
K(L)= & \int\left(\frac{\partial \boldsymbol{\beta}}{\partial L}\right)^{T} V^{-1}(\boldsymbol{y}-\boldsymbol{\beta}(L))(\boldsymbol{y}-\boldsymbol{\beta}(L))^{T} \\
& \times V^{-1}\left(\frac{\partial \boldsymbol{\beta}}{\partial L}\right) p(\boldsymbol{y}, L) d \boldsymbol{y} \\
= & \left(\frac{\partial \boldsymbol{\beta}}{\partial L}\right)^{T} V^{-1}\left(\frac{\partial \boldsymbol{\beta}}{\partial L}\right)
\end{aligned}
$$

It is seen from the equations (11) and (12) that the value of Fisher's information matrix corresponds to the sharpness of the peak of the likelihood function.

The advantage of the introduction of the sharpness of the likelihood function as the criterion is that we can select desirable data window and modes from the viewpoint of not only the peak sharpness but also the shape of the likelihood function.

That is, it is well-known that all modes of the stationary wave have frequencies of integer multiples of the frequency of the 1st mode. Therefore, the likelihood function has essentially a single peak around the true value. But, when phase shifts occur on the stationary waves, the frequencies of the sound fluctuation of the pipe change and thus the peak of the likelihood function is split into some peaks. Moreover, in the case where phase lead and phase lag happen to cancel to each other, the shape of the peak of the likelihood function takes a gentle slope.

Consequently, the optimal data window and the optimal modes are obtained by selecting the window and the modes which has a single sharp peak (around the true length) on the likelihood function. Applying the optimal data window and the modes to the measurement method mentioned in section 2, a high accurate mesurement of pipe is achieved.

\section{Experiments}

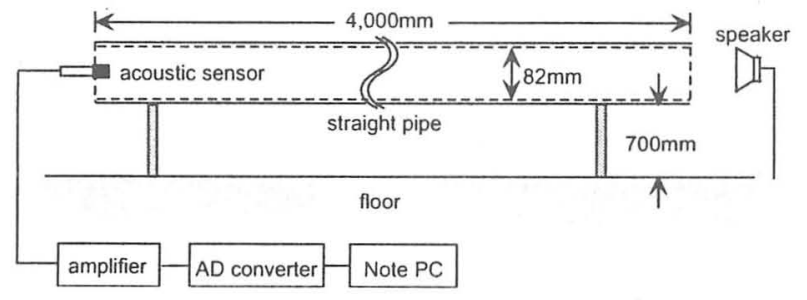

Fig. 2 Outline of the experiment.

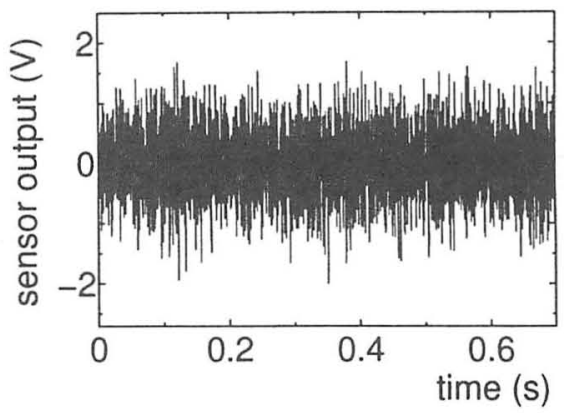

Fig. 3 Sensor output under a continual external noise.

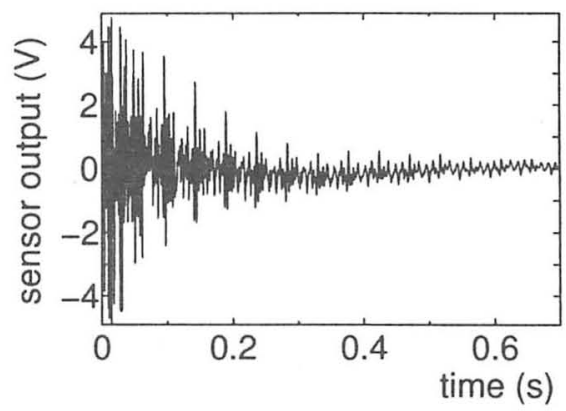

Fig. 4 Sensor output under no external noise.

Fig. 2 shows the outline of the experiment. The length and diameter of the pipe are $4,000 \mathrm{~mm}$ and $82 \mathrm{~mm}$, respectively. The temperature in the pipe at the experiment was $21.5^{\circ} \mathrm{C}$. The noise was generated by a speaker set outside the pipe at another side of the sensor. The sound fluctuation in the pipe was observed with a capacitor type microphone and memorized by Note PC for the duration of 10 s with the sampling period $\Delta T=0.2 \mathrm{~ms}$. The sensor output is shown in Fig.3, for reference. We see that the sound fluctuation is strongly disturbed by the noise. This can be easily recognized, if we see the sound fluctuation in the pipe under no external noise shown in Fig.4. Fig.4 shows the sound fluctuation generated in the pipe by a shock with a club. In the figure, periodical large peaks are clearly observed, which reflect the fundamental mode of the stationary waves in the pipe. For reference, we show in Figs.5 and 6 respectively the frequency spec-

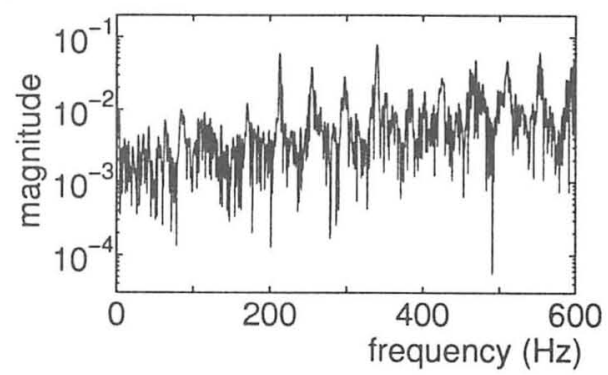

Fig. 5 Spectrum of the sensor output under the continual noise. 


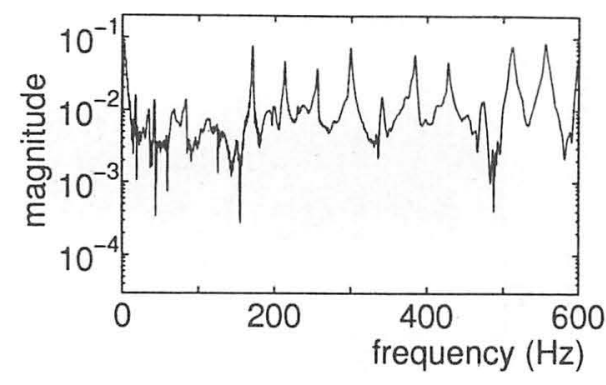

Fig. 6 Spectrum of the sensor output under no noise except the hitting of the pipe.

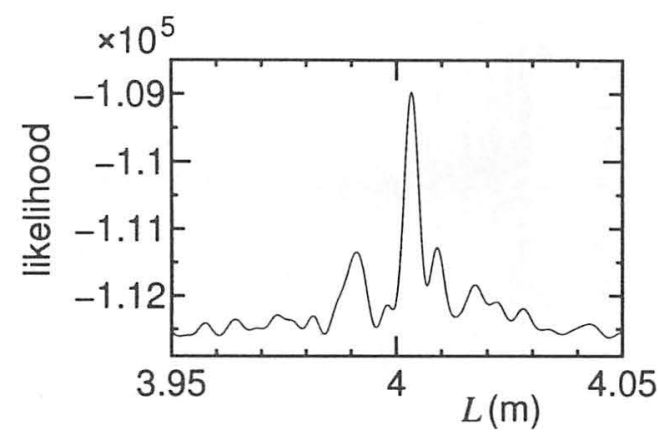

Fig. 7 A likelihood function for the optimal data window and the optimal modes.

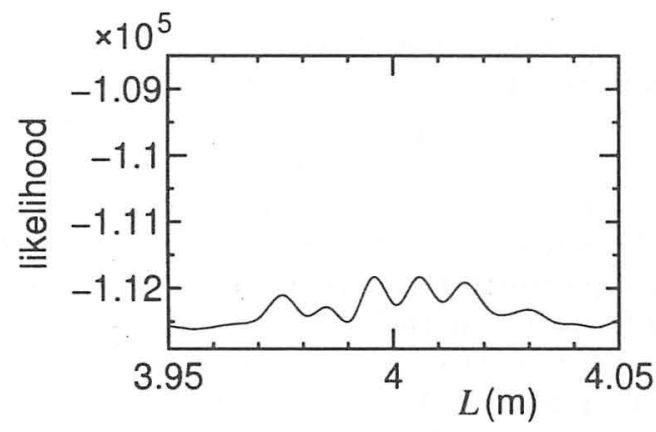

Fig. 8 A likelihood function for a data window $[0,2.0]$ and its optimal modes.

tra of the sensor outputs. The figures show us that it is difficult for the stationary waves to stand in the pipe under the continual external noise.

The length of the data window is now set as $2 \mathrm{~s}$ and the starting time of the data window was changed little by little by $0.1 \mathrm{~s}$. For each data window, the optimal modes were sought and then the sharpness of the peak of the logarithmic likelihood function was evaluated. The finally selected data window and the optimal modes were given by $[6.9,8.9]$ and $\{5,8,13\}$, respectively.

For reference, Fig. 7 shows the logarithmic likelihood function for the selected optimal data window and the optimal modes, whereas Fig. 8 shows that for the data window $[0,2.0]$ and its optimal modes. From the figures, we see that the proposed method is highly evaluated.
Table'1. Measurement results.

\begin{tabular}{|l|r|r|}
\hline & mean $(\mathrm{mm})$ & SD $(\mathrm{mm})$ \\
\hline true & 4,000 & \\
\hline \hline proposed method & 4,002 & 3.7 \\
\hline pre-proposed method & 4,004 & 14.6 \\
\hline \multicolumn{3}{|c|}{ SD : standard deviation }
\end{tabular}

Applying the measurement system in section 2 under the optimal data window and the optimal modes, the measurment result of $L$ is obtained as $L=4.003 \mathrm{~m}$.

For 10 times experiments, the measurement results by the proposed method became like in Table 1 . The table also shows, for reference, the measurement results by the pre-proposed method when it was applied to the data window $[0,2.0]$. The table concludes that a much higher accurate pipe length measurement is achieved with the proposed method compared to the pre-proposed one.

\section{Conclusions}

The authors previously proposed an accurate pipe length measurement system using stationary waves in the pipe in a quiet environment. The paper proposed a length measurement system in the case where the stationary waves are continually disturbed by external noises, considering the situation where the length measurement of the pipe with open ends is required under a noisy environment.

For realizing it, the sharpness of the peak of the likelihood function was adopted as a criterion and the optimal data window and the optimal modes to get rid of the noise affect were selected to apply the previous method.

Experiments showed that the proposed method realized a highly accurate pipe length measurement even in a noisy environment.

\section{References}

[1] S.Tanaka and M.Okamoto: Dynamic Model Based Pipe Length Measurement Using Stationary Wave, Measurement, Vol.28, 249/259 (2000)

[2] S.Tanaka and M.Okamoto : Highly Accurate Pipe Length Measurement for Straight Pipes with Open Ends Using Stationary Waves, Journal of The Society of Instrument and Control Engineers, Vol.38,No.10,1/8 (2002)

[3] T.Igarashi : Acoustics and Vibrations, Kyoritsu Syuppan (1968)

[4] S.Arimoto : Kalman Filter, Sangyo-Tosho (1977)

[5] S.Kullback: Information Theory and Statistics, John Wiley and Sons (1959) 